\title{
Nonlinear RF-Driven Sheared Flows in Toroidal Geometry
}

\author{
J. R. Myra and D. A. D'Ippolito \\ Lodestar Research Corporation, Boulder, Colorado, USA
}

\begin{abstract}
The modification of background plasma flows in a tokamak by applied rf waves is of interest as a possible means of controlling turbulence, triggering the formation of internal transport barriers. Here we extend previous work by developing the standard neoclassical treatment to enable i) an evaluation of localized (in radius) poloidal and toroidal forces by the rf, ii) a rigorous calculation of the resulting poloidal and toroidal flow rates in the presence of neoclassical damping due to plasma viscosity and a phenomenological radial diffusion of momentum, and iii) implicit treatment of ambipolarity issues in the presence of rf. As an example, we consider IBW interaction within a narrow resonant ion-cyclotron layer where the wave absorption and rf forces occur.
\end{abstract}

\section{INTRODUCTION}

Nonlinear forces arising from applied rf waves are of interest for the generation of bulk plasma flows. Because sufficiently large rf-driven sheared flows can in principle suppress turbulence, it has been proposed that rf power may be useful as an external control for triggering tokamak plasma transitions into high-confinement regimes. Indeed there is intriguing experimental evidence for this in several IBW experiments.

Our goal in the present paper is to put previous slab model sheared-flow drive work [1-3] on a firm footing with respect to toroidal geometry and ambipolarity considerations. This necessarily entails a neoclassical approach to the problem. Our treatment follows most closely the procedure in Refs. 4 and 5; however, here we explore further the coupling of toroidal and poloidal flows in the presence of a phenomenological radial diffusion of momentum. The latter effect is expected to be important for localized (in radius) poloidal and toroidal rf forces, and also adds significantly to the conceptual understanding of the resulting flows. The toroidal formulation provides an implicit treatment of ambipolarity issues in the presence of rf.

As in Ref. 3, the driving rf forces are due to the localized absorption of rf waves in a radially narrow resonance layer in which both plasma Larmor radius effects and dissipation play important roles. The resulting forces and flows are bipolar in nature, (i.e. changing sign across the resonance layer) when the net momentum imparted by the waves to the plasma is small; however, significant sheared flows, suitable for turbulence suppression, can still arise. Furthermore, when momentum diffusion dominates damping, net bulk plasma rotation can arise even when the wave momentum input is negligible. This result is in analogy to a similar situation in which fast particles rather than rf nonlinearities are the momentum source [6]. 


\section{TOROIDAL FORMULATION}

Beginning with the momentum conservation equation in vector form, including a phenomenological diffusion coefficient $\mathrm{D}$, we take toroidal and parallel components to obtain the equations

$$
\begin{gathered}
-\frac{\mathrm{D}}{v} \frac{\partial}{\partial \psi} v\left\langle\mathrm{R}^{2} \mathrm{~B}_{\theta}^{2} \frac{\partial}{\partial \psi} \rho \mathrm{Ru}_{\zeta}\right\rangle=\left\langle\mathrm{RF}_{\zeta}\right\rangle \\
-\frac{\mathrm{D}}{v} \frac{\partial}{\partial \psi} v\left\langle\mathrm{R}^{2} \mathrm{~B}_{\theta}^{2} \frac{\partial}{\partial \psi} \rho \mathrm{Bu}_{\|}\right\rangle+\langle\mathbf{B} \cdot \nabla \cdot \Pi\rangle=\left\langle\mathrm{BF}_{\|}\right\rangle
\end{gathered}
$$

where $\rho=\mathrm{nm}_{\mathrm{i}}, \psi$ is a radial flux coordinate, $\langle>$ is a flux surface average, $v$ is a Jacobian factor, and $\theta$ and $\zeta$ are the poloidal and toroidal angles respectively. In Eqs. (1) and (2), F is the total nonlinear force due to the rf waves (including the important contributions from the rf pressure tensor discussed in Refs. [2] and [3]), and $\Pi$ is the neoclassical contribution that will give rise to the damping of poloidal flows. Thus $\mathrm{F}$, given explicitly in Ref. 3, is a generalization of the simple $\mathrm{rf}$ ponderomotive force.

Equations (1) and (2) are standard (see e.g. Refs. [4, 5]), except for the retention of D in Eq. (2), which will be critical. Since $\langle\mathbf{B} \cdot \nabla \cdot \Pi\rangle$ is proportional to the poloidal flow velocity, Eq. (2) would seem to suggest that the poloidal flow is driven by the parallel component of $\mathrm{F}$; however, this is not the case, even when $\mathrm{D} \rightarrow 0$.

Assuming incompressible bulk flows in toroidal geometry, we employ the standard form for the velocity $\mathbf{u}=\mathrm{K}(\psi) \mathbf{B}+\mathrm{G}(\psi) \mathbf{R}_{\zeta}$ with $\mathrm{K}$ and $\mathrm{G}$ to be determined. From radial force balance, $\mathrm{G}$ is related to the radial gradients and the radial rf force by

$$
\mathrm{G}=-\mathrm{c}\left(\frac{\partial \Phi}{\partial \psi}+\frac{1}{\operatorname{Zen}} \frac{\partial \mathrm{p}_{\mathrm{i}}}{\partial \psi}\right)+\frac{\mathrm{c}}{\mathrm{Zen}_{\mathrm{i}}} \frac{\mathrm{F}_{\mathrm{i} \psi}}{\mathrm{RB}_{\theta}} .
$$

Thus, the rf affects the ambipolar radial electric field $E_{r}$ by $\left.i\right)$ the toroidal rotation $G$ it drives through $\mathrm{F}_{\zeta}$, ii) the $\mathrm{p}_{\mathrm{i}}(\psi)$ heating profile for fixed $\mathrm{G}$, and iii) the explicit radial force $\mathrm{F}_{\mathrm{i} \psi}$. Normally, the latter effect is negligible; but if not, the rf can also drive flux surface distortions [7].

The equations for $\mathrm{K}$ and $\mathrm{G}$ can be simplified and decoupled in several limits. In the case of localized responses (sharp gradients in $\mathrm{G}$ and $\mathrm{K}$ relative to the plasma radius) the equation for $\mathrm{K}$ becomes

$$
\left[\left\langle\mathrm{B}^{2}\right\rangle_{\mathrm{x}}-\frac{\mathrm{R}^{2} \mathrm{~B}_{\zeta}^{2}}{\left\langle\mathrm{R}^{2}\right\rangle_{\mathrm{x}}}\right] \mathrm{K}^{\prime \prime}-\frac{3 \eta_{0}}{\mathrm{D} \rho}\left\langle\left(\nabla_{\|} \mathrm{B}\right)^{2}\right\rangle \mathrm{K}=-\frac{\left\langle\mathrm{BF}_{\|}\right\rangle}{\mathrm{D} \rho}+\frac{\mathrm{RB} \zeta\left\langle\mathrm{RF}_{\zeta}\right\rangle}{\mathrm{D} \rho\left\langle\mathrm{R}^{2}\right\rangle_{\mathrm{x}}}
$$

where $x$ is a local radial variable, ${ }^{\prime}=\mathrm{d} / \mathrm{dx},\langle\rangle_{\mathrm{x}}$ is a weighted flux surface average whose detailed form is unimportant here, and we have specialized to the PfirschSchlüter limit with $\eta_{0}$ the classical parallel ion viscosity. Note that when $\mathrm{D} \rightarrow 0, \mathrm{Eq}$. (4) does not recover Eq. (2) with $\mathrm{D}=0$. Equation (1) implies that $\mathrm{G} \propto 1 / \mathrm{D}$ as $\mathrm{D} \rightarrow 0$. Thus, the diffusion term in Eq. (2), $\mathrm{Du} \| \propto \mathrm{DG}$, is not negligible as $\mathrm{D} \rightarrow 0$. Equation (4) recovers the heuristic result that the poloidal flow $\mathrm{K}$ is driven by a force component which is essentially poloidal, i.e. the rhs of Eq. (4). Equation (4) also permits a rigorous calculation of forced flows in the presence of damping.

In the case of small $\varepsilon=\mathrm{r} / \mathrm{R}_{0}$, circular flux surface geometry, for general collisionality (banana, plateau or Pfirsch-Schlüter) we obtain the uncoupled equations

$$
\mathrm{D} \nabla_{\mathrm{r}}^{2}(\hat{\mathrm{J}})=-\mathrm{F}_{\zeta}
$$




$$
\mathrm{D} \nabla_{\mathrm{r}}^{2}\left[\mathrm{~b}_{\theta}^{2} \hat{\mathrm{K}}\left(1+2 \mathrm{q}^{2}\right)\right]-\Gamma \hat{\mathrm{K}}=-\mathrm{b}_{\theta} \mathrm{F}_{\theta}
$$

where $\quad \mathbf{b}=\mathbf{B} / \mathrm{B}, \quad \hat{\mathrm{J}}=\rho \mathrm{GR}_{0}\left(1+3 \varepsilon^{2} / 2\right)+\rho \mathrm{KB}_{\zeta_{0}}=\rho \mathrm{u}_{\zeta}, \quad \hat{\mathrm{K}}=\rho \mathrm{KB}=\rho \mathrm{u}_{\theta} / \mathrm{b}_{\theta}$, $\Gamma \hat{\mathrm{KB}}=<\mathbf{B} \cdot \nabla \cdot \Pi>$. Here, $\nabla_{\mathrm{r}}^{2}$ is the usual cylindrical Laplace operator.

It is straightforward to investigate Eqs. (5) and (6) in various limiting cases of the scale lengths of the rf absorption layer $\Delta$, the diffusion scale $\lambda=\left[D\left(1+2 q^{2}\right) / \gamma_{\theta}\right]^{1 / 2}$ and the minor radius a. The main results are as follows. For $\mathrm{D} \rightarrow 0$, i.e. $\lambda<<\Delta$, we obtain $\mathrm{u}_{\theta}=\mathrm{F}_{\theta} / \gamma_{\theta} \rho$ where in the collisional regime, $\gamma_{\theta}=3 \mathrm{v}_{\mathrm{i}}^{2} / 2 \mathrm{R}^{2} \mathrm{v}_{\mathrm{ii}}$ differs from the standard expression [8] by $\mathrm{R}^{2} \rightarrow \mathrm{R}^{2}\left(1+2 \mathrm{q}^{2}\right)$. The standard result can be recovered from the present theory when the time derivatives are retained and the unforced initial value problem is solved. For $\Delta<<\lambda<<$ a, the local poloidal $\mathrm{rf}$ force drives local poloidal flows while the local toroidal rf force drives both local and global toroidal flows. In the strong diffusion limit, [Eq. (5) for toroidal or Eq. (6) with $\Delta<<\lambda \sim$ a for poloidal] one can drive net flows with no direct rf momentum input when the boundary conditions at $\mathrm{r}=0$ and $\mathrm{r}=$ a provide symmetry-breaking [6].

\section{NUMERICAL EXAMPLE}

The nonlinear force density on a fluid element due to second order interactions in the rf electric field strength has been calculated in considerable generality in Ref. 3 . The calculations are fully electromagnetic, and include contributions from the hot plasma Bessel function terms, and the resulting rf-induced pressure tensor. While only the poloidal force component was required in Ref. 3, the toroidal force may readily be obtained from the expressions therein. The force components of Ref. 3 are related to those required here by $\mathbf{e}_{\mathrm{x}}=\mathbf{e}_{\psi}, \mathbf{e}_{\mathrm{z}}=\mathbf{b}$ and $\mathbf{e}_{\mathrm{y}}=\mathbf{b} \times \mathbf{e}_{\psi}$. Two classes of poloidal and toroidal terms arise: those corresponding to direct momentum input by the waves (proportional to $\mathrm{k}_{\theta}$ and $\mathrm{k}_{\zeta}$ respectively) and those corresponding to a conservative redistribution of momentum (and hence perfect $\partial / \partial x$ derivatives).

As an illustration, results for a test case similar to one treated in Ref. 2 are presented: that of IBW absorption in a cyclotron layer. We consider a TFTR-like tritium plasma, take $\mathrm{k}_{\mathrm{y}}=0$, and define $\mathrm{x}$ so that the $\omega=5 \Omega_{\mathrm{t}}$ resonance is located at $\mathrm{x}$ $=0$. A one-dimensional eikonal-based algorithm is employed to obtain the electric field profile near the resonance layer, retaining the full Bessel and Z-function ion response. Figure 1a) shows the Poynting flux and absorbed power for a case with total power absorption $\sim 400 \mathrm{~kW}$. The electric field $\mathrm{E}_{\mathrm{X}} \sim 100 \mathrm{~V} / \mathrm{cm}$ (not shown) undergoes about 12 oscillations between $\mathrm{x}=5$ and $2 \mathrm{~cm}$ before its amplitude becomes negligible, justifying the eikonal limit. Figure 1b) shows the nonlinear force and resulting poloidal flow for the parameters $\gamma_{\theta}=10 / \mathrm{s}$ and $\mathrm{D}=200 \mathrm{~cm}^{2} / \mathrm{s}$. Note the bipolar nature of $F_{\theta}$ and the diffusive broadening of $u_{\theta}$ relative to $F_{\theta}$. The peak value of $u_{\theta} \approx 2 \mathrm{~km} / \mathrm{s}$ (for this assumed D) is of the same order as that measured experimentally in TFTR [9] and the profile shape shows qualitative agreement with the experiment. The calculated toroidal flow velocity profile for this example (not shown) is $\approx 30 \mathrm{~km} / \mathrm{s}$ at $\mathrm{r}$ $=0$ and is constant out to the resonance layer $(\mathrm{r}=46)$ after which it decays slowly to zero at $\mathrm{r}=\mathrm{a}$. It is driven primarily by the net toroidal momentum of the waves, which were taken to have an asymmetric spectrum with $\mathrm{k}_{\mathrm{z}} \sim 5 \mathrm{~m}^{-1}$.

The poloidal flow for this example is consistent with the estimate $\mathrm{u}_{\theta}=$ $\mathrm{P}_{\mathrm{rf}} \Delta / 4 \mathrm{q}^{2} \Omega \mathrm{D} \rho \mathrm{V}$ with the $\mathrm{P}_{\mathrm{rf}}$ the total rf power and the relevant volume $\mathrm{V}=4 \pi^{2} \mathrm{Rr} \Delta$. For the toroidal flow, the estimate is $\mathrm{u}_{\zeta}=\mathrm{P}_{\mathrm{rf}} \mathrm{k}_{\zeta} \mathrm{r}^{2} / \omega \mathrm{D} \rho \mathrm{V}$ where since the toroidal flows are global $\mathrm{V}=2 \pi^{2} \mathrm{Rr}^{2}$. In units of $\mathrm{km} / \mathrm{s}, \mathrm{MW}, \mathrm{m}, \mathrm{MHz}, \mathrm{T}, \mathrm{m}^{2} / \mathrm{s}$, and $10^{20} / \mathrm{m}^{3}$ we obtain $\mathrm{u}_{\theta}=0.4 \mathrm{P}_{\mathrm{rf}} / \mathrm{q}^{2} \mathrm{RrBDn}_{20}$ and $\mathrm{u}_{\zeta}=48 \mathrm{P}_{\mathrm{rf}} \mathrm{k}_{\zeta} / \mathrm{RfDn}_{20} \mu$ with $\mu(\mathrm{amu})$ the ion mass and $\mathrm{f}(\mathrm{MHz})$ the rf frequency. 

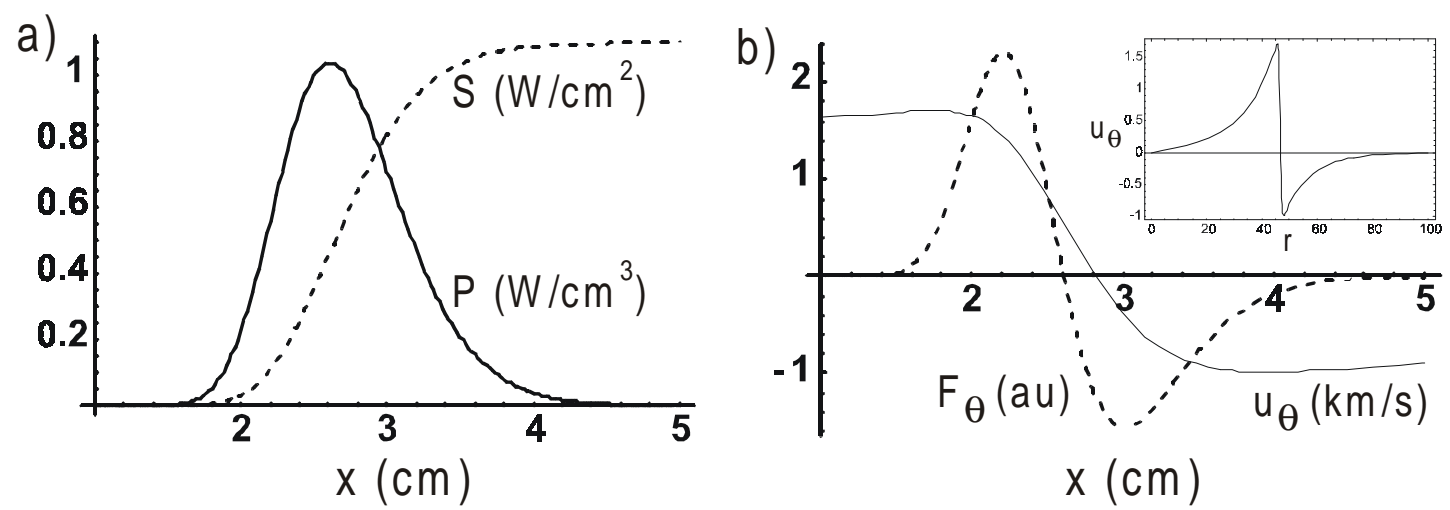

FIGURE 1. a) Power absorption $P\left(W / \mathrm{cm}^{3}\right)$ (solid) and Poynting flux $S\left(W / \mathrm{cm}^{2}\right)$ (dashed) for IBW absorption near a cyclotron resonance layer $(\mathrm{x}=0), \mathrm{b})$ resulting force $\mathrm{F}_{\theta}$ (arbitrary units) (dashed) and poloidal flow velocity $\mathrm{u}_{\theta}(\mathrm{km} / \mathrm{s})$ (solid). The inset shows $\mathrm{u}_{\theta}$ over the full range of minor radius.

\section{CONCLUSIONS}

A formulation of rf-driven flows in toroidal geometry has been developed which includes effects arising from the geometry and from $\mathrm{rf}$ ambipolarity. It was shown that retaining diffusion in the parallel momentum equation (as well as in the toroidal equation) is critical, and results in $u_{\theta}$ being driven by $F_{\theta}$ rather than $F_{\|}$. The results enable a rigorous calculation of forced poloidal flows in the presence of damping by viscosity. In the Pfirsch-Schlüter regime the result differs slightly from the often quoted damping rate for the decay of unforced flow. When diffusion is strong, localized rf forces can drive net global flows even without direct wave momentum input. Finally, the flows appear to be of a significant size for case of directly launched IBW, and are in qualitative agreement with experimental data.

\section{ACKNOWLEDGMENTS}

This work was supported by the U.S. Department of Energy under Grant DE-FG0397ER54392. The authors grateful acknowledge discussions with E.F. Jaeger.

\section{REFERENCES}

1. G.G. Craddock and P.H. Diamond, Phys. Rev. Lett. 67, 1535 (1991).

2. E.F. Jaeger et al., Phys. Plasmas 7, 3319 (2000).

3. J.R. Myra and D.A. D'Ippolito, Phys. Plasmas 7, 3600 (2000).

4. Y.R. Lin-Liu, V.S. Chan and S.C. Chiu in 11th Topical Conference on Radio Frequency Power in Plasmas, Palm Springs, CA, 1995 (American Institute of Physics, Melville, 1996), p. 247.

5. V.S. Chan, S.C. Chiu, Y.R. Lin-Liu and Y.A. Omelchenko in 13th Topical Conference on Radio Frequency Power in Plasmas, Annapolis, MD, 1999 (American Institute of Physics, Melville, 1999), p. 45; and refs. therein.

6. F.W. Perkins, R.B. White, P.T. Bonoli and V.S. Chan, Phys. Plasmas 8, 2181 (2001).

7. J.R. Myra, Phys. Fluids 31, 1190 (1988) and refs. therein.

8. A.B. Hassam and R.M. Kulsrud, Phys. Fluids 21, 2271 (1978).

9. J.R. Wilson, R.E. Bell, S. Bernabei, K. Hill, et al., Phys. Plasmas 5, 1721 (1998). 